\title{
Performance of transported PDF mixing models in a turbulent premixed flame
}

\author{
Michael Kuron ${ }^{\mathrm{a} *}$, Evatt Hawkes ${ }^{\mathrm{b}}$, Zhuyin Ren $^{\mathrm{c}}$, Joshua Tang ${ }^{\mathrm{b}}$, Hua Zhou ${ }^{\mathrm{c}}$, \\ Jacqueline H. Chen ${ }^{\mathrm{d}}$, Tianfeng $\mathrm{Lu}^{\mathrm{a}}$ \\ ${ }^{a}$ Department of Mechanical Engineering, University of Connecticut, Storrs, CT 06269, USA \\ ${ }^{\mathrm{b}}$ School of Photovoltaic and Renewable Energy Engineering, The University of New South Wales, \\ Sydney, NSW 2052, Australia \\ ${ }^{\mathrm{c}}$ Center for Combustion Energy and School of Aerospace Engineering, Tsinghua University, Beijing \\ 100084, China \\ ${ }^{\mathrm{d}}$ Combustion Research Facility, Sandia National Laboratories, Livermore, CA 96551, USA
}

*Corresponding Author:

Michael Kuron

University of Connecticut, Department of Mechanical Engineering

191 Auditorium Road U-3139, Storrs, CT 06269-3139, USA

michael.kuron@uconn.edu

Colloquium: Turbulent Flames

Total Length of Paper: 6155 (Determined using Method 1)

- Main Text: 3749 words

- Equations: 68 words

- References: 385 words

- Figures and Captions: 1953 words in total

$\circ$ Figure 1: 345 words Figure 2: 465 words Figure 3: 174 words

○ Figure 4: 310 words Figure 5: 186 words Figure 6: 473 words

Supplemental material is available for this submission.

All figures are to be printed in black and white 


\begin{abstract}
Modeling of premixed turbulent flames is challenging due to the effects of strong turbulencechemistry interaction. In the transported probability density function (TPDF) methods, chemical reactions are treated exactly, while molecular mixing needs to be modeled. In the present study, the performance of three widely used mixing models, namely the Interaction by Exchange with the Mean (IEM), Modified Curl (MC), and Euclidean Minimum Spanning Tree (EMST) models, are assessed using direct numerical simulation (DNS) data of a lean premixed hydrogen-air slot jet flame simulated at Sandia. The DNS provides initial conditions and time varying input quantities, including the mean velocity, turbulent diffusion coefficient, and scalar mixing rate for the TPDF simulations. A number of progress variable definitions are explored, as well as the commonly used constant mechanical-to-scalar mixing timescale model. It is found that the EMST model provides the best prediction of the flame structure and flame propagation speed out of the models tested. The IEM model implies a qualitatively incorrect conditional mean and RMS diffusion rate, while the MC model can qualitatively capture the conditional mean diffusion rate. Only the EMST model can accurately predict the conditional mean diffusion rate for this flame, which can be attributed to its enforcement of mixing that is local in composition space. Finally, a parametric study on the mechanical-to-scalar timescale ratio is performed. It is found that the optimal choice for the timescale ratio varies by a factor of 2 for the two DNS cases study, despite the cases having the same configuration. Therefore, this commonly used approach does not appear to be viable for turbulent premixed flames and further attention to mixing timescale models for reactive scalars is merited.
\end{abstract}

Keywords: turbulent premixed flames, direct numerical simulation, transported probability density function, micro-mixing models 


\section{Introduction}

Lean turbulent premixed combustion offers compelling advantages for modern engine design due to limited pollutant emissions and high fuel efficiencies. However, turbulent premixed combustion involves complex flame dynamics and turbulence-chemistry interactions that are challenging to model in the Reynolds-averaged Navier-Stokes (RANS) and Large Eddy Simulations (LES). In particular, the transported probability density function (TPDF) method is an extensively studied modeling approach where challenges remain when being applied for turbulent premixed flames $[1,2]$. Specifically, while the nonlinear chemical source term appears in closed form in the TPDF approach, the molecular mixing, or micro-mixing, term is unclosed, and modeling of the molecular mixing term is the primary challenge in the TPDF methods $[3,4]$.

Mixing models such as the Interaction by Exchange with the Mean (IEM) [5], Modified Curl (MC) [6,7], and Euclidean Minimum Spanning Tree (EMST) [8] are widely adopted in the TPDF methods. The ability of the TPDF method to capture complex phenomena such as extinction and re-ignition has been well demonstrated for non-premixed flames for a variety of experimental and computational configurations $[2,9]$. In contrast, fewer studies have been performed using the TPDF method in the context of premixed flames. Due to the complex coupling of molecular mixing and reaction in premixed flames, the accuracy of the molecular mixing models is critical for the overall performance of TPDF and it is necessary to systematically assess the performance of these mixing models for simulating premixed combustion. Several studies have been performed [10-12] for this purpose. While it has been posited that the mixing model is the primary source of error [3], it is not clear whether the error is due to the mixing model formulation or other modelled features, such as the scalar mixing rate.

In the present study, direct numerical simulation (DNS) of a temporally evolving premixed HydrogenAir slot jet [13] is used as a numerical test bed to evaluate the IEM, MC and EMST mixing models in the context of turbulent premixed flames. The DNS database is used to supply the initial conditions and all 
time varying inputs to the governing equations in the composition TPDF method, including the mean velocity, turbulent diffusion coefficient, and mixing rate. The same thermodynamic properties and chemical reaction mechanism used in the DNS are used in the TPDF simulations to limit the potential sources of modeling error to the mixing model. The aim of this study is to rigorously benchmark the performance of the three mixing models using DNS data to assess their suitability for turbulent premixed flames. The effects of different progress variable definitions on the model performance, as well as the conditional diffusion and turbulent flame speed implied by each mixing model, are explored. Furthermore, in practical implementations of the TPDF methods, the mixing rate is often modelled as being linearly proportional to the turbulent time scale with a constant coefficient $C_{\phi}$, which must be chosen a priori and is an approach founded on the basis of passive scalar mixing. The viability of using a constant $C_{\phi}$ for reactive scalar mixing is also evaluated in the present study.

\section{Analysis Methodology}

\subsection{DNS Configuration}

The 3-D DNS dataset of a lean $\mathrm{H}_{2}$-air turbulent premixed flame with detailed chemistry in [13] is used as the basis for the TPDF simulations. The configuration is described in detail in [13] and is only summarized here (see Fig. S1 in the supplemental material for a diagram of the DNS configuration). In the simulation, two initially planar flames propagate into a slab of fresh reactants in the domain center, with strong turbulence sustained by the mean shear. The flames begin to interact with the shear layer after the $11^{\text {th }}$ jet time and the sheared turbulence is fully developed after 14 jet times, with the maximum flame wrinkling occurring near the $17^{\text {th }}$ jet time. The temperature of the unburned reactants is $700 \mathrm{~K}$, the background pressure is atmospheric, and the equivalence ratio is $\phi=0.7$. The physical domain is $16 \mathrm{H} \mathrm{x}$ $20 \mathrm{H} x 12 \mathrm{H}$ in the $\mathrm{x}, \mathrm{y}$, and $\mathrm{z}$ directions, respectively, where $\mathrm{H}$ is the initial fuel jet thickness. The boundaries in the streamwise $(\mathrm{x})$ and spanwise $(\mathrm{z})$ directions are periodic while the boundaries in the transverse direction (y) are treated as non-reflecting outflows. The simulation was performed using the 
Sandia DNS code, S3D [14]. A detailed chemical kinetic model of hydrogen oxidation with 9 species and 21 reversible chemical reactions was employed [15]. The DNS configuration allows for RANS averaging in the streamwise and spanwise directions, as well as the use of symmetry across the domain centerline in the transverse direction. After averaging, the simulation is reduced to statistical dependence on the transverse direction and time.

We consider here the two cases, namely "Da" and "Da" as shown in Fig. S1, where case $\mathrm{Da}^{-}$has $\mathrm{Da}<1$ and case $\mathrm{Da}^{+}$has $\mathrm{Da}>1$ throughout the entire simulation. Based on the non-dimensional flame parameters, both cases are in the thin reaction zones regime.

\subsection{TPDF Solution Methodology}

The TPDF solver has been implemented using a hybrid particle-mesh methodology in the RANS context as in a previous study of mixing models in non-premixed flames by Krisman et. al. [9]. A 1-D Eulerian mesh is created with the extents in the transverse direction of half of the DNS domain on account of the symmetry about the centerline. The Eulerian mesh is populated with notional Lagrangian particles which obey the set of governing stochastic differential equations, Eqs. 1 and 2 [2,9], which solve for transport in physical and composition space:

$$
\begin{gathered}
d x^{*}(t)=\left[\widetilde{V}+\frac{\nabla \tilde{\Gamma}_{t} \bar{\rho}}{\bar{\rho}}\right] d t+\left(2 \tilde{\Gamma}_{t}\right)^{1 / 2} d W \\
d \phi^{*}(t)=[\mathrm{m}] \mathrm{dt}+S\left(\phi^{*}\right) d t
\end{gathered}
$$

where $x^{*}$ and $\boldsymbol{\phi}^{*}$ are the spatial location and composition, respectively, of a particle, $\tilde{V}$ is the mean transverse velocity, $\tilde{\Gamma}_{t}$ is the turbulent diffusion coefficient, $d W$ is the increment of a Wiener process, $\mathrm{S}\left(\boldsymbol{\phi}^{*}\right)$ is the chemical source term, and $[\mathrm{m}]$ is the rate of change in composition due to mixing. Note that molecular diffusion is ignored in Eq. 1 as it is found that the turbulent diffusion coefficient is larger than the molecular diffusion coefficient by at least an order of magnitude throughout the flame brush.

The mean transverse velocity and turbulent diffusivity are both extracted directly from the DNS results at each time step and fed to the TPDF solver. The turbulent diffusion coefficient is computed as 
$\tilde{\Gamma}_{t}=\frac{|\widetilde{\rho} c-\bar{\rho} \bar{v} \tilde{c}|}{\bar{\rho}|\nabla \tilde{c}|}$ where $v$ is the velocity, $\rho$ is the density, and $\mathrm{c}$ is the progress variable [2]. Additionally, each mixing model requires as an input a mixing rate, which is defined as $\Omega=\widetilde{\chi_{c}} / \widetilde{c^{12}}$ where $\widetilde{\chi_{c}}$ is the Favre-averaged scalar dissipation rate of the progress variable and $\widetilde{c^{12}}$ is the Favre-averaged variance of the progress variable [2]. The scalar dissipation rate is computed as $\widetilde{\chi_{c}}=2 D_{c} \nabla \overline{c^{\prime \prime} \nabla c}$, where $D_{c}$ is the diffusivity of the progress variable. As with the velocity and turbulent diffusion coefficient, the mixing rate is extracted from the DNS results at each time step and supplied to the TPDF solver.

For turbulent premixed flames with strong differential molecular diffusion effects, the scalar dissipation rate is sensitive to the choice of progress variable and therefore its potential impact on flame propagation and structure are also investigated in this study, the results of which are shown in the next section. The progress variable defined by a reactant species is computed as $c=\left(Y_{R}-Y_{R, u}\right) /\left(Y_{R, b}-Y_{R, u}\right)$, while the progress variable defined by a product species is computed as $c=Y_{P} / Y_{P, b}$, where the subscripts $\mathrm{u}$ and $\mathrm{b}$ refer to the unburnt and equilibrium states, respectively.

All input data required by the TPDF solver has been computed by Favre-averaging the DNS data in the statistically homogeneous $\mathrm{x}$ and $\mathrm{z}$ directions at each time step. The simulations are initialized from the DNS data at the $11^{\text {th }}$ jet time, which is when the flame begins to interact with the shear layer.

\subsection{Micro-Mixing Models}

The features and limitations of the three mixing models compared in this study have been extensively discussed in the literature [5-8]. The IEM model is deterministic and forces the particle compositions to linearly relax to the local mean. The MC model is a stochastic model which randomly pairs particles within a cell whose compositions instantaneously change to an intermediate state. The EMST model is a stochastic model that organizes the particles within a cell into a minimum spanning tree such that pairwise mixing only occurs between particles that are nearest neighbors in composition space. The IEM and MC models are both simplistic in nature. For instance, the IEM model is only strictly valid for passive scalars 
in a statistically homogenous Gaussian field [16]. The MC model places no restrictions on which particles in a cell can mix, so in practice a particle can be mixed across a strongly burning flame front [17]. The EMST model seeks to rectify these shortcomings by enforcing that mixing is local in composition space. This property ensures that only particles of similar states can be mixed, which is expected to be beneficial for premixed flames where strong coupling between mixing and reaction are present [18]. Note that in a study of these three mixing models for non-premixed flames [9], it was suggested that the simpler IEM and MC models should suffice for flames without significant local extinction, while the EMST model should be used in more challenging conditions.

In addition to the mixing format, the mixing rate plays a central role in all three mixing models. The mixing rate is often modelled as being linearly proportional to the turbulent time scale with a constant $C_{\phi}$, which is called into question in turbulent premixed flames where micro-mixing of reactive scalars is governed by both turbulence and chemical reactions $[3,10]$.

\section{Results and Discussion}

\subsection{Convergence Study}

To minimize the influence of numerical errors, a convergence study is performed to determine a suitable level of spatial and temporal resolution. The key numerical parameters are the number of Eulerian cells, $N_{\text {cell }}$; number of particles per cell, $N_{p c}$; and the time step used to integrate the TPDF equations, $t_{\text {step }}$. The convergence study has been performed using Case $\mathrm{Da}^{-}$and the IEM mixing model with the progress variable defined based on the mass fraction of $\mathrm{H}_{2}$.

The sensitivity of the RMS temperature profiles to the key numerical parameters is shown in Figure 1. Since the RMS values are expected to converge more slowly than the mean values, only the RMS is reproduced here. The convergence of the mean values can be found in Fig. S2. In the top row of the figure the sensitivity to the number of Eulerian cells is shown to converge quickly as the number of cells increases. The solutions for variable number of cells are generated using 4,000 particles per cell and an 
integration time step of $2.5 \mathrm{~ns}$. The sensitivity of the solution to the number of particles per cell, shown in the bottom row of Figure 1, is generated using 300 cells and an integration time step of 2.5 ns. The results demonstrate that the smoothness of the solution profiles is most sensitive to the number of particles per cell and that the solutions reach a sufficient level of smoothness at 4,000 particles per cell.

Based on the behavior observed in this convergence study, all results in this paper for Case $\mathrm{Da}^{-}$are generated using 300 cells, 4,000 particles per cell, and a time step of 5 ns. A similar study has been performed for Case $\mathrm{Da}^{+}$, resulting in the numerical parameters of 600 cells, 4,000 particles per cell, and a time step of $10 \mathrm{~ns}$. It is noted that the TPDF solver used in this study does not perform any smoothing, and thus the convergence rate is slow and requires significantly more particles. If smoothing were to be applied, the convergence rate would be much faster, and a particle level of 20-100 particles per cell, as found elsewhere in the literature, could be expected to achieve a converged solution [19].

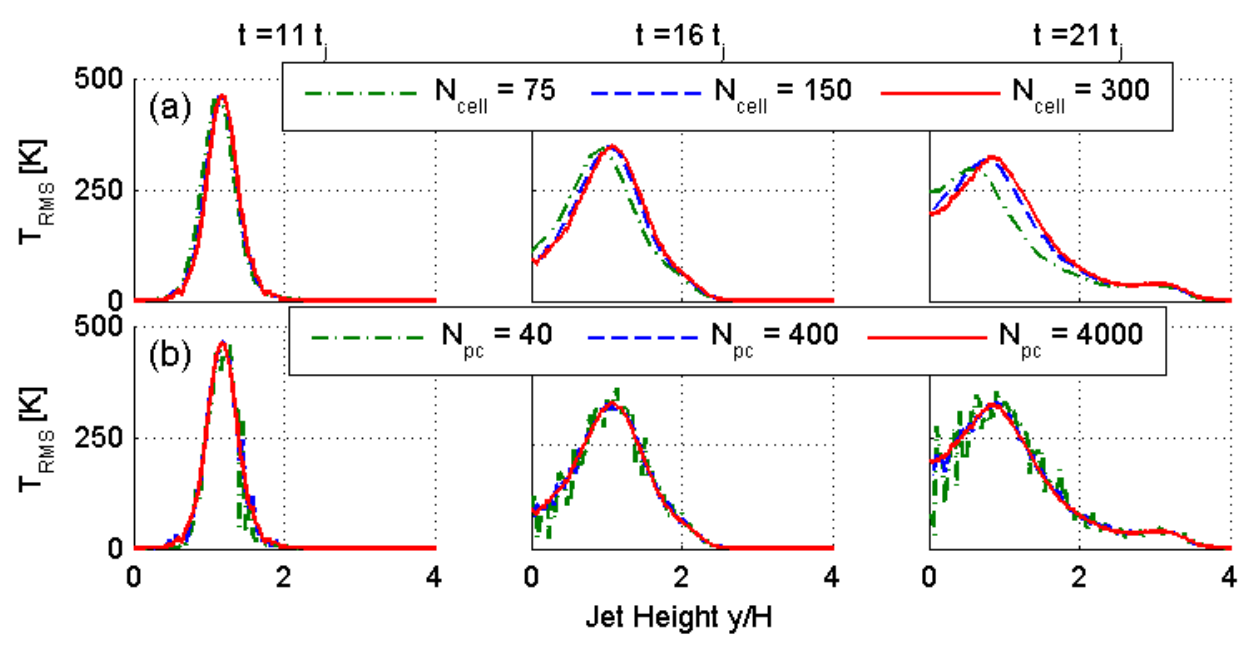

Figure 1 - Solution convergence behavior with varying numerical parameters. Row (a): variable number of cells. Row (b): variable number of particles per cell.

\subsection{Effects of Mixing Models on Flame Propagation and Flame Structure}

Due to the strong coupling between molecular mixing and reaction in premixed flames, the mixing model performance can have significant effects on TPDF solutions. The mixing model directly affects the 
evolution of the RMS scalar values, which in turn can affect the mean scalar values through the reaction term. The temporal evolution of the mean and RMS temperature, computed using a progress variable defined on the mass fraction of $\mathrm{H}_{2}$ for both DNS cases, are shown in Figure 2.

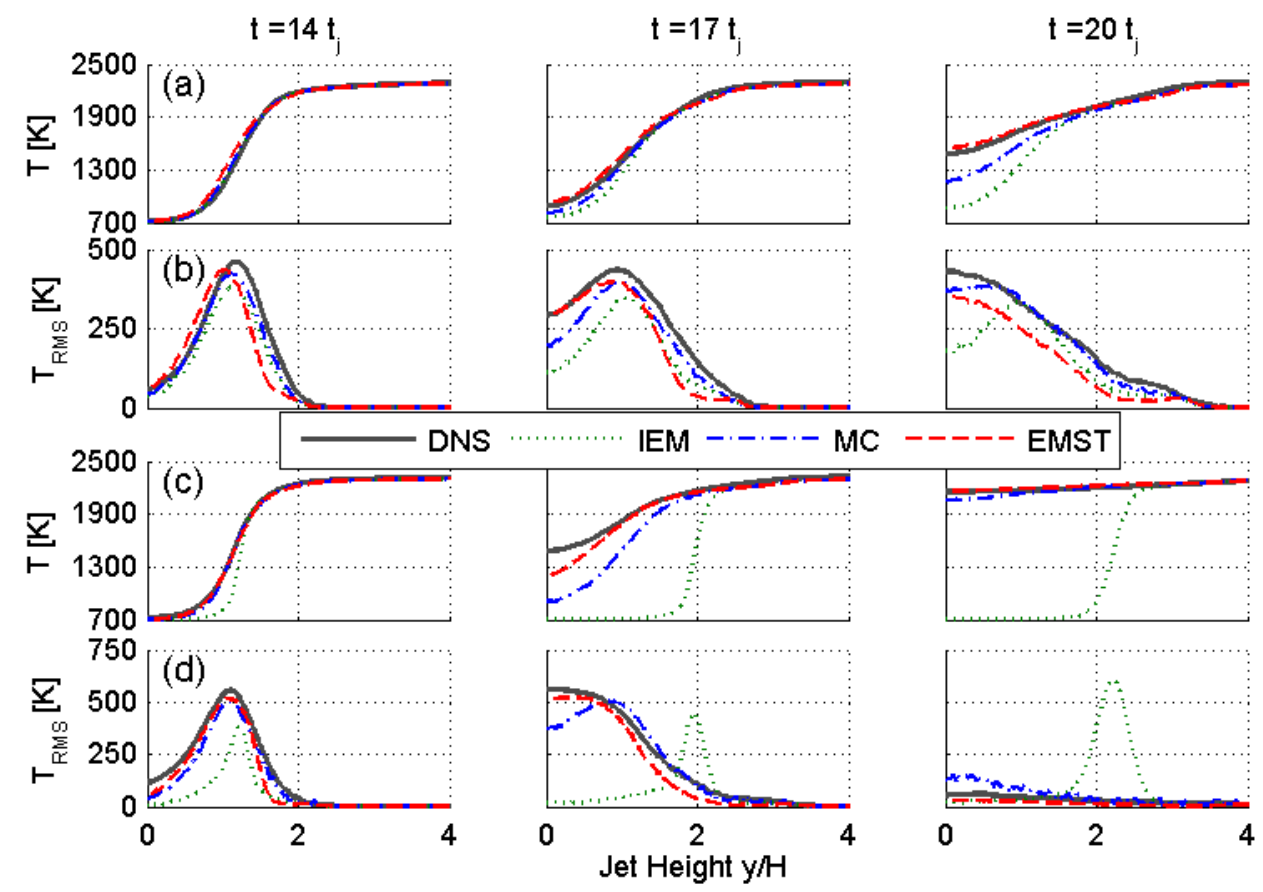

Figure 2 - Comparison of mixing model performance at different times using the progress variable defined on $\mathrm{H}_{2}$ mass fraction. Rows (a) and (b): Case $\mathrm{Da}^{-}$. Rows (c) and (d): Case $\mathrm{Da}^{+}$.

In both cases, the IEM model under-predicts the propagation of the mean flame profile and provides a qualitatively incorrect prediction of the spatial RMS profiles. The MC model provides better predictions of the mean flame structure than the IEM model, but still tends to under-predict the mean flame propagation speed. Similar to the IEM model, the MC model is non-local in composition space, resulting in difficulty in maintaining the correct scalar profiles. In contrast, the EMST mixing model demonstrates the best performance among the three models. It is seen that the mean DNS spatial temperature profiles are closely tracked by the EMST model, and the RMS temperature profiles are captured reasonably well for both cases. The superior performance of the EMST model can be attributed to its ability to enforce mixing that is local in composition space. This feature allows the EMST model to 
reasonably approximate the flame structure while the IEM and MC models fail (see Fig. S3 to S8 for the flame structure predictions). To further quantify the effect of the mixing models on flame propagation, the turbulent flame speed, $\mathrm{S}_{\mathrm{T}}$, defined based on the global consumption rate (for comparison with the DNS data) is constructed as:

$$
S_{\mathrm{T}}=\frac{1}{\rho_{\mathrm{u}}} \int_{0}^{\mathrm{ymax}} \overline{\dot{\omega}_{\mathrm{c}}}(y) \mathrm{dy}
$$

where $\overline{\dot{\omega}_{c}}$ is the mean mass production rate of the progress variable defined based on $\mathrm{H}_{2}$, and $\rho_{u}$ is the density in the unburnt reactants. As shown in Fig. 3, the EMST model provides the best prediction of the flame speed variation for both cases. The MC model is reasonably accurate for Case $\mathrm{Da}^{-}$, but is less accurate in the more flamelet-like Case $\mathrm{Da}^{+}$, while the IEM model fails for both cases. In case $\mathrm{Da}^{-}$, the flame speed for the EMST model deviates from the DNS early in the simulation. The deviation can be attributed to the EMST mixing model over-predicting the mass fractions of the radical species during the early stages of the simulation, making the mixture in the EMST results more reactive, on average. Thus the flame tends to propagate more quickly initially, before becoming more closely aligned with the DNS data for this case.

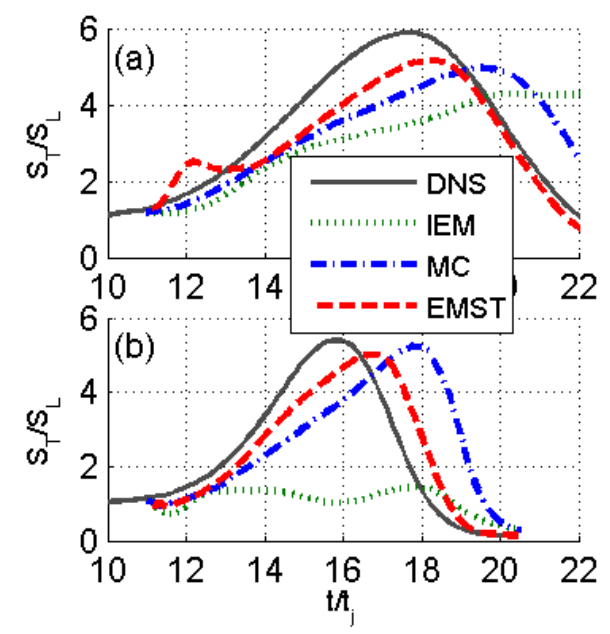

Figure 3 - Turbulent flame speed vs. time. (a) Case $\mathrm{Da}^{-}$. (b) Case $\mathrm{Da}^{+}$

\subsection{Differential Diffusion Effects}


For the hydrogen flame considered here, the scalar dissipation rate, and thus the mixing rate, is sensitive to the choice of progress variable due to strong differential diffusion effects. Tests on progress variables defined based on temperature and mass fractions of $\mathrm{H}_{2}, \mathrm{O}_{2}, \mathrm{H}_{2} \mathrm{O}$ and $\mathrm{OH}$ show that the mixing rates computed from $\mathrm{H}_{2}$ and $\mathrm{O}_{2}$ bound the solution space for this flame. As demonstrated in Figure 4, the TPDF solution from the EMST model is sensitive to the progress variable definition, particularly in the more flamelet-like Case $\mathrm{Da}^{+}$. Similar sensitivity is observed for the IEM and MC models as well. The progress variable definition can therefore affect the TPDF solution through the mixing rate magnitude and spatial profile, as shown in Figs. S9 and S10. It is seen that mixing rate based on the deficient reactant, $\mathrm{H}_{2}$, most closely tracks the results from the DNS, whereas the mixing rate based on $\mathrm{O}_{2}$ results is the least accurate.

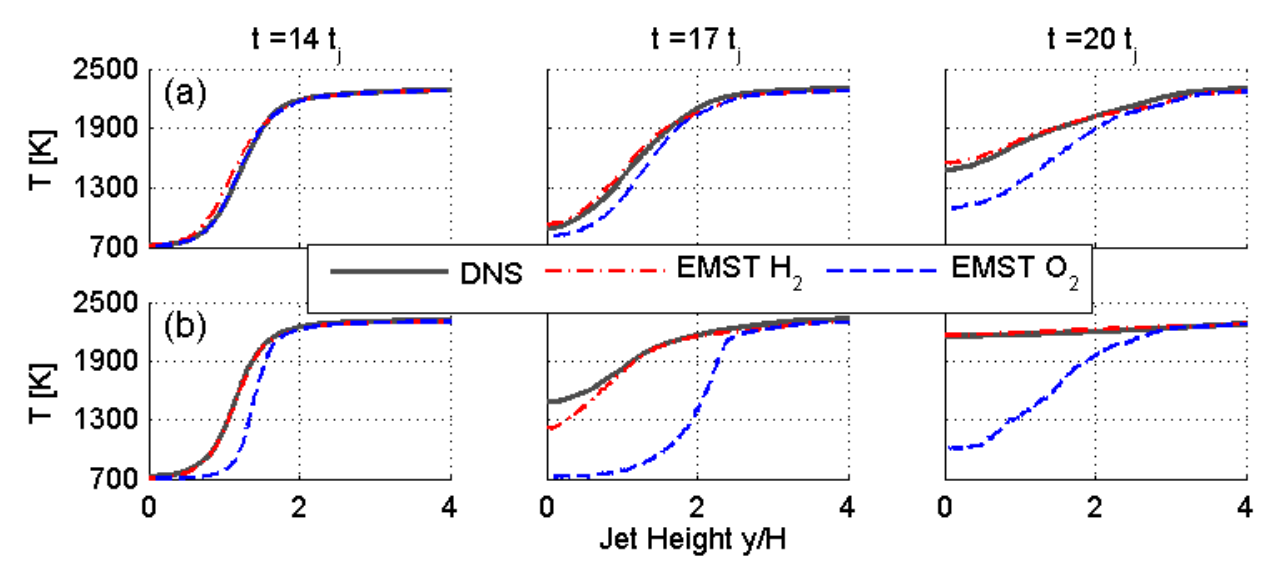

Figure 4 - Evolution of mean temperature from the TPDF solutions with EMST for different progress variable definitions. Row (a): Case $\mathrm{Da}^{-}$. Row (b): Case $\mathrm{Da}^{+}$

The significant difference in the mixing rate can be partially attributed to the difference in molecular weight, indicating the important role that differential diffusion plays in this flame. Note that in the present test, all scalars are assumed to mix over the same timescale in the mixing models which renders the results sensitive to the definition of the progress variable. This effect is expected to be weaker for hydrocarbon flames where differential diffusion effects are less important.

\subsection{Conditional Diffusion}


The aim of each mixing model is to accurately mimic the conditional diffusion using the methods described in section 2.3. To evaluate the ability of each mixing model to mimic the conditional diffusion rate, we have extracted the diffusion rates conditionally averaged on the progress variable that are implied by each mixing model in the TPDF simulations for comparison to the DNS. The conditional mean diffusion rates are extracted from the DNS data by first computing the diffusion rate of each species at each grid point in the DNS domain at each time point using mixture-averaged transport properties. Then, the conditional mean diffusion rates for a given time point are computed by conditionally averaging the diffusion rate of each species on the progress variable. The conditional mean diffusion rate implied by each mixing model is computed in the TPDF solution by storing the incremental change in the composition vector due only to mixing over a given time step for each particle. The incremental change in composition due to mixing is divided by the time step size to compute the implied diffusion rate for each species in each particle, and the particle diffusion rates are then conditionally averaged on the progress variable to compute the conditional mean diffusion rate for each mixing model at each time point.

The conditional mean diffusion rates of $\mathrm{H}_{2}$ implied by each mixing model for the TPDF simulations, based on the progress variable defined on $\mathrm{H}_{2}$, are shown in Figure 5 for both cases at time $14 \mathrm{t}_{\mathrm{j}}$. It is seen that the IEM model fails to capture the correct conditional mean diffusion rate entirely, while the MC model qualitatively captures the conditional mean diffusion rate behavior but over-predicts the diffusion rate on the burned side of the flame. This inaccuracy in the diffusion rate can be attributed to the nonlocality and inherent randomness of the model, which in principle allows for particles to be mixed across strongly burning flame fronts. The EMST model most accurately captures the conditional diffusion rate, implying that EMST has the correct mixing format in composition space for a turbulent premixed flame. 


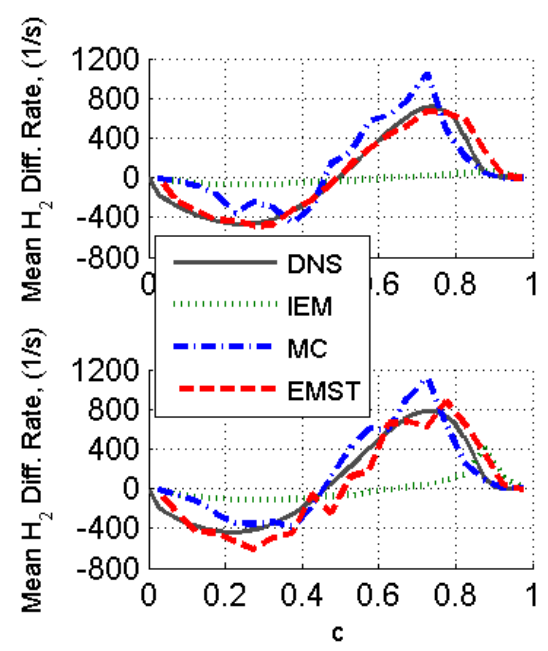

Figure 5 - Conditional mean diffusion rates for Case $\mathrm{Da}^{-}$(top) and Case $\mathrm{Da}^{+}$(bottom) at $14 \mathrm{t}_{\mathrm{j}}$.

\subsection{The Mixing Rate Model}

In practical TPDF calculations the scalar mixing rate is not known a priori and must be modeled. In many applications the mixing rate is assumed to be inversely proportional to the turbulent timescale, $\Omega=$ $C_{\phi} \Omega_{\mathrm{t}}=C_{\phi} \tilde{\varepsilon} / \tilde{k}$, where $\Omega_{\mathrm{t}}$ is the turbulence mixing rate and can be reconstructed based on the turbulence dissipation rate, $\tilde{\varepsilon}$, and the turbulence kinetic energy, $\tilde{k}$, in the RANS context. The constant $C_{\phi}$ is typically assumed to be 2.0 for passive scalar mixing, although several DNS studies have shown that the optimal choice even for non-premixed flames is unclear [2]. For premixed flames, where the scalar mixing timescale is also influenced by reaction, it is questionable whether a constant mechanical-to-scalar timescale ratio is applicable. To address this question, the turbulent mixing rate is extracted from the DNS in the same manner as the scalar mixing rate and a parametric study is performed on the timescale ratio.

Figure 6 shows the effect of the mechanical-to-scalar timescale ratio on the TPDF solution at $17 \mathrm{tj}$ for both cases, after the sheared turbulence has developed from the initial conditions. For Case $\mathrm{Da}^{-}$the results indicate that the optimal value for $C_{\phi}$ is between 2 and 4 , while for Case $\mathrm{Da}^{+}$the optimal value for $C_{\phi}$ is between 4 and 6 . The spatial and temporal distribution of $C_{\phi}$ is extracted from the DNS using the mixing rate based on $\mathrm{H}_{2}$ and plotted in Figure S11. The local value of the timescale constant varies by up to an 
order of magnitude. It is therefore clear that there is no single value of the mechanical-to-scalar timescale ratio that is optimal for all cases, and variation in the optimal choice of the $C_{\phi}$ parameter between these two cases by a factor of 2 makes the turbulent timescale a poor choice for the mixing rate. Additionally, the RMS profiles are qualitatively incorrect in the middle of the flame brush for both cases, indicating that the spatial distribution of the turbulent mixing rate is inconsistent with the spatial distribution of the scalar mixing rate.
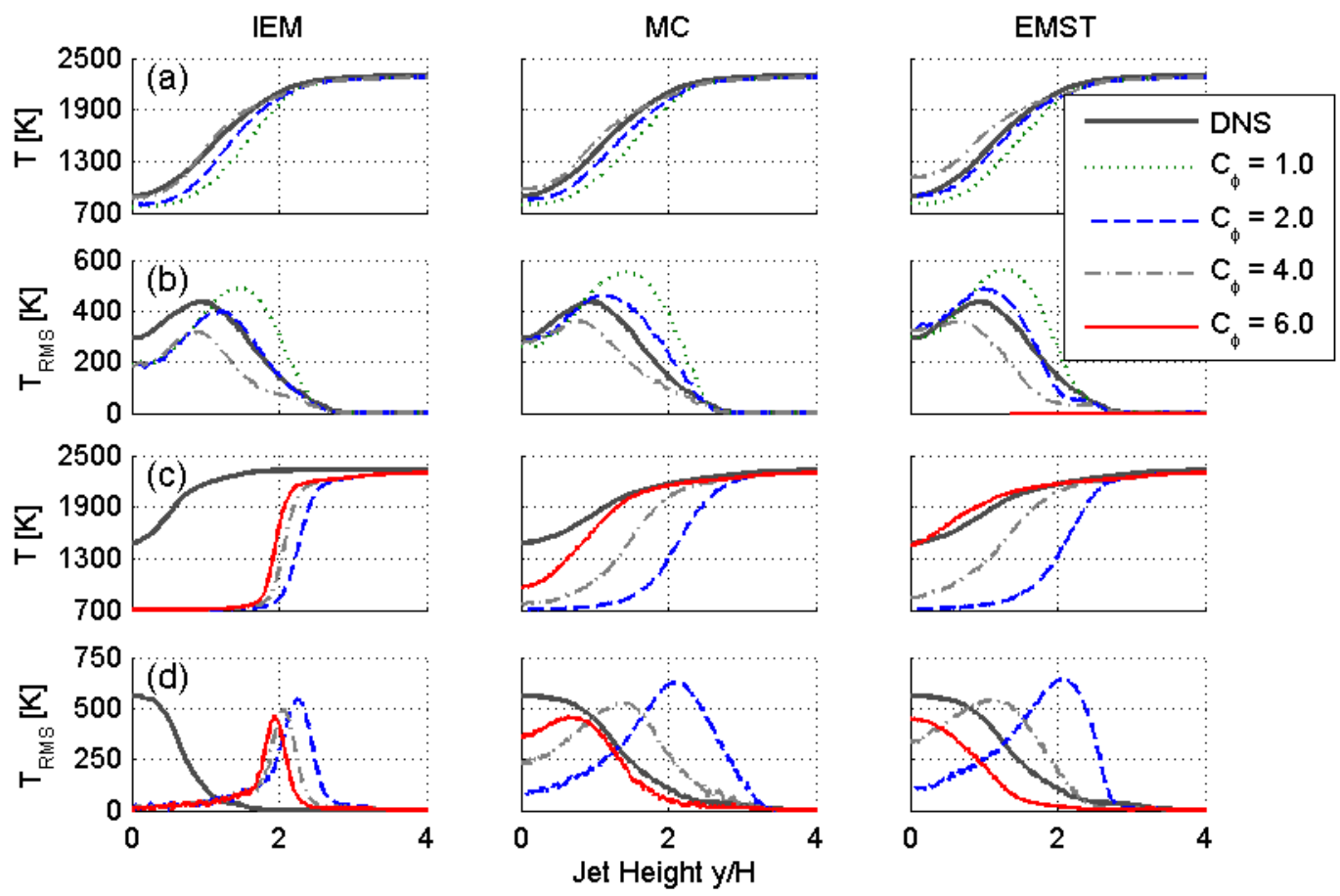

Figure 6 - Influence of the mechanical-to-scalar timescale ratio on the TPDF solution at $\mathrm{t}=17 \mathrm{tj}$. Rows (a) and (b): Case $\mathrm{Da}^{-}$. Rows (c) and (d): Case $\mathrm{Da}^{+}$.

\section{Conclusions}

Three widely adopted mixing models are evaluated in the present study in the context of the RANS composition TPDF method using DNS data of a turbulent premixed $\mathrm{H}_{2}$-air flame. The methodology employed here allows for the quantification of the errors in mixing model formulations incurred in the TPDF solution. 
Direct comparison of the TPDF predictions of the mean and RMS values with those from the DNS reveals that the EMST mixing model provides the most accurate solutions for the present flame, and that the EMST model can accurately capture the conditional mean diffusion rates. The conditional diffusion implied by the IEM model is qualitatively incorrect for turbulent premixed flames. The MC model is able to qualitatively capture the mean conditional diffusion. The better performance of EMST compared with IEM and MC can be attributed to the local mixing in composition space.

The commonly used method of specifying the mixing rate by assuming that it is inversely proportional to the turbulence timescale with a constant mechanical-to-scalar timescale ratio is examined. It is found that the TPDF solutions are sensitive to the mechanical-to-scalar timescale ratio, and that the optimal values for the two DNS cases differ by a factor of two. Given that such a wide variation in the optimal choice of the mechanical-to-scalar timescale ratio exists even for a flame in the same configuration, this commonly used approach does not appear to be viable for turbulent premixed flames. The development of accurate timescale models for reactive scalar mixing merits further study. In particular, future development of the joint pdf of the composition and its gradients may provide a more suitable approach for capturing spatial structure governed by molecular diffusion affected by reaction [20].

\section{Acknowledgements}

This work was supported by the National Science Foundation and the Department of Energy through the NSF-DOE Partnership on Advanced Combustion Engines Program under Grant CBET-1258646. The work at Sandia was supported by the Division of Chemical Sciences, Geosciences and Bio-sciences, the Office of Basic Energy Sciences, the U.S. Department of Energy. Sandia National Laboratories is a multiprogram laboratory operated by Sandia Corporation, a Lockheed Martin Company, for the U.S. Department of Energy under contract DE-AC04-94-AL85000. This material is based upon work supported by the U.S. Department of Energy, Office of Science, Office of Workforce Development for Teachers and Scientists, Office of Science Graduate Student Research (SCGSR) program. The SCGSR program is 
administered by the Oak Ridge Institute for Science and Education for the DOE under contract number DE-AC05-06OR23100. The work at Tsinghua University was supported by National Natural Science Foundation of China (91441202).

\section{References}

[1] S.B. Pope, Prog. Energy Combust. Sci. 11 (1985) 119-192.

[2] D.C. Haworth, Prog. Energy Combust. Sci. 36 (2010) 168-259.

[3] D.H. Rowinski, S.B. Pope, Combust. Theory Mod. 15 (2011) 245-266.

[4] S. Mitarai, J.J. Riley, G. Kosály, Phys. Fluids. 17 (2005) 047101.

[5] J.C.D. J. Villermaux, Proc. 2nd Int. Symp. on Chem. Reaction Eng. (1972) 1-13.

[6] R.L. Curl, AIChE J. 9 (1963) 175-181.

[7] J. Janicka, W. Kolbe, W. Kollmann, J. Non Equilib. Thermodyn. 4 (1979) 47-66.

[8] S. Subramaniam, S.B. Pope, Combust. Flame. 115 (1998) 487-514.

[9] A. Krisman, J.C.K. Tang, E.R. Hawkes, D.O. Lignell, J.H. Chen, Combust. Flame. 161 (2014) 1-22.

[10] D.H. Rowinski, S.B. Pope, Phys. Fluids. 25 (2013) 105105.

[11] M. Stöllinger, S. Heinz, Combust. Flame. 157 (2010) 1671-1685.

[12] R.P. Lindstedt, E.M. Vaos, Combust. Flame. 145 (2006) 495-511.

[13] E.R. Hawkes, O. Chatakonda, H. Kolla, A.R. Kerstein, J.H. Chen, Combust. Flame. 159 (2012) 2690-2703.

[14] J.H. Chen, A. Choudhary, B. de Supinski, M. DeVries, E.R. Hawkes, S. Klasky, W.K. Liao, K.L. Ma, J. Mellor-Crummey, N. Podhorszki, R. Sankaran, S. Shende, C.S. Yoo, Comp. Sci. Disc. 2 (2009) 015001.

[15] J. Li, Z. Zhao, A. Kazakov, F.L. Dryer, Int J Chem Kinet. 36 (2004) 566-575.

[16] S.B. Pope, Turbulent Flows, Cambridge University Press, 2000.

[17] Z. Ren, S.B. Pope, Combust. Flame. 136 (2004) 208-216. 
[18] S.B. Pope, Proc. Combust. Inst. 34 (2013) 1-31.

[19] S. Viswanathan, H. Wang, S.B. Pope, Journ. Comp. Phys. 230 (2011) 6916-6957.

[20] S.B. Pope, Symp. Int. Combust. 23 (1991) 591-612. 


\section{List of Figures and Captions:}

Figure 1 - Solution convergence behavior with varying numerical parameters. Row (a): variable number of cells. Row (b): variable number of particles per cell. ............................................... 8

Figure 2 - Comparison of mixing model performance at different times using the progress variable defined on $\mathrm{H}_{2}$ mass fraction. Rows (a) and (b): Case $\mathrm{Da}^{-}$. Rows (c) and (d): Case $\mathrm{Da}^{+} \ldots \ldots \ldots \ldots . . . .9$

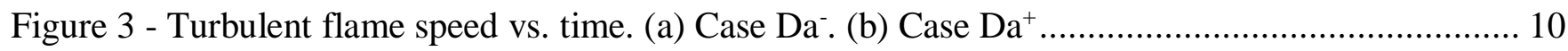

Figure 4 - Evolution of mean temperature from the TPDF solutions with EMST for different progress

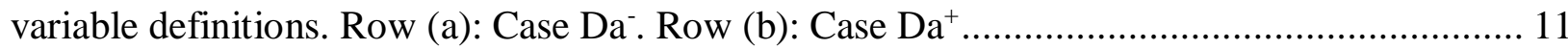

Figure 5 - Conditional mean diffusion rates for Case $\mathrm{Da}^{-}$(top) and Case $\mathrm{Da}^{+}$(bottom) at $14 \mathrm{t}_{\mathrm{j} . . . \ldots \ldots \ldots . . . . .13}$

Figure 6 - Influence of the mechanical-to-scalar timescale ratio on the TPDF solution at $\mathrm{t}=17 \mathrm{tj}$. Rows

(a) and (b): Case $\mathrm{Da}^{-}$. Rows (c) and (d): Case $\mathrm{Da}^{+}$. 